Journal for ImmunoTherapy of Cancer

\section{Identification of dual positive CD19+/ CD3 + T cells in a leukapheresis product undergoing CAR transduction: a case report}

To cite: Schultz L, Patel S, Davis KL, et al. Identification of dual positive CD19+/CD3+ T cells in a leukapheresis product undergoing CAR transduction: a case report. Journal for ImmunoTherapy of Cancer 2020;8:e001073. doi:10.1136/ jitc-2020-001073

- Additional material is published online only. To view please visit the journal online (http://dx.doi.org/10.1136/jitc2020-001073).

Accepted 12 August 2020

Check for updates

(c) Author(s) (or their employer(s)) 2020. Re-use permitted under CC BY-NC. No commercial re-use. See rights and permissions. Published by BMJ.

${ }^{1}$ Pediatrics, Stanford University School of Medicine, Stanford, California, USA

${ }^{2}$ Stanford University, Stanford Cancer Institute, Stanford California, USA

${ }^{3}$ Pediatrics, Lucile Packard Children's Hospital, Palo Alto, California, USA

Correspondence to

Liora Schultz;

liora.schultz@gmail.com

\section{ABSTRACT}

Background Chimeric antigen receptor (CAR) therapy and hematopoietic stem cell transplantation (HSCT) are therapeutics for relapsed acute lymphocytic leukemia (ALL) that are increasingly being used in tandem. We identified a non-physiologic CD19+/CD3+ T-cell population in the leukapheresis product of a patient undergoing CAR T-cell manufacturing who previously received a haploidentical HSCT, followed by infusion of a genetically engineered T-cell addback product. We confirm and report the origin of these CD19+/CD3+ T cells that have not previously been described in context of CAR T-cell manufacturing. We additionally interrogate the fate of these CD19-expressing cells as they undergo transduction to express CD19-specific CARs.

Main body We describe the case of a preteen male with multiply relapsed B-ALL who was treated with sequential cellular therapies. He received an $\alpha \beta$ T-cell depleted haploidentical HSCT followed by addback of donor-derived T cells genetically modified with a suicide gene for iCaspase 9 and truncated CD19 for cell tracking (RivoCel). He relapsed 6 months following HSCT and underwent leukapheresis and CAR T-cell manufacturing. During manufacturing, we identified an aberrant T-cell population dually expressing CD19 and CD3. We hypothesized that these cells were RivoCel cells and confirmed using flow cytometry and PCR that the identified cells were in fact RivoCel cells and were eliminated with iCaspase 9 activation. We additionally tracked these cells through CD19-specific CAR transduction and notably did not detect T cells dually positive for CD19 and CD19-directed CARs. The most likely rationale for this is in vitro fratricide of the CD19+ 'artificial' T-cell population by the CD19-specific CAR+ T cells in culture.

Conclusions We report the identification of CD19+/ CD3+ cells in an apheresis product undergoing CAR transduction derived from a patient previously treated with a haploidentical transplant followed by RivoCel addback. We aim to bring attention to this cell phenotype that may be recognized with greater frequency as CAR therapy and engineered $\alpha \beta$ haplo-HSCT are increasingly coupled. We additionally suggest consideration towards using alternative markers to CD19 as a synthetic identifier for post-transplant addback products, as CD19-expression on effector T cells may complicate subsequent treatment using CD19-directed therapy.

\section{BACKGROUND}

Chimeric antigen receptor (CAR) $\mathrm{T}$ cells have demonstrated remarkable effect in patients with refractory/relapsed B-acute lymphoblastic leukemia (B-ALL). ${ }^{1-5}$ Patient outcomes drastically vary, driven partly by interpatient heterogeneity of the composition of each individual CAR T-cell product. Efforts to characterize CAR T cell products are vital to advancing the field. ${ }^{46}$ Here we describe the identification of an aberrant T-cell population expressing surface CD19 that was identified during CAR manufacturing.

As the repertoire of antileukemia therapies expands to include a spectrum of genetically engineered targeted therapies, leukemias that were historically fatal can become 'chronic'. These therapies can however drive the biology of both $\mathrm{B}$ and $\mathrm{T}$ cells to artificially depart from their native baseline. Following CAR T-cell therapy, many patients receive consolidative hematopoietic stem cell transplantation (HSCT). The available stem cell donor pool recently expanded to include alternative donors (ie, HLA-haploidentical donors), sometimes combined with post-HSCT addbacks, including genetically engineered donorderived cellular products. One such example that shows striking promise is the $\alpha \beta$ T-cell/ CD19 B-cell depleted haploidentical HSCT, followed by an infusion of donor-derived $\mathrm{T}$ cells genetically modified with a safety gene for iCaspase9 (RivoCel). Inclusion of the suicide gene, commonly introduced using a gammaretroviral vector, allows for elimination of the modified T cells on infusion of the dimerizing agent AP1903, in case of uncontrolled graft-versus-host disease (GvHD). ${ }^{8}{ }^{9}$ The described RivoCel add back $\mathrm{T}$ cells are additionally engineered to express truncated 
CD19 as a marker to permit cell-tracking. ${ }^{10}$ In receiving combinations of HSCT and genetically modified cellular therapies, we are building new artificial immune systems.

Here, we report the identification of an unexpected dual expressing CD19+/CD3+ cell subset found in a patient who underwent work-up for CAR T-cell therapy following a haploidentical HSCT. We aim to bring attention to this cell phenotype that may be recognized with greater frequency as CAR therapy and haploidentical HSCTs are increasingly coupled.

\section{CASE PRESENTATION}

We report the case of a preteen male with a history of multiply relapsed B cell precursor-ALL with unremarkable cytogenetics, who was treated with sequential cellular therapy products. His initial relapse was an isolated bone marrow relapse over 1 year from completing upfront maintenance chemotherapy and was managed with chemotherapy as per BFM95. Three months following chemotherapy completion, he experienced a second relapse with combined CNS and bone marrow disease and was treated with commercial CD19-specific CAR T-cell therapy, Tisagenlecleucel (Kymriah). He tolerated therapy with only Grade I CRS and achieved a minimal residual disease negative remission by next generation sequencing by day 28 following infusion. Due to his history of multiple relapses, he proceeded to HSCT preemptively, 3 months following Tisagenlecleucel. Due to lack of either related or unrelated matched donors, the patient was enrolled in a phase II clinical trial (NCT03301168) and received $\alpha \beta$ T-cell/CD19 B-cell depleted haploidentical HSCT followed by an addback of donor T cells genetically modified to express an iCas9 inducible suicide vector (RivoCel). The post-transplant period was uneventful. Six months after transplantation, the patient developed new onset back pain and was found to be pancytopenic with peripheral blasts. A bone marrow aspirate at that time confirmed the presence of $>90 \%$ blasts preserving expression of CD19. In context of an early post-transplant relapse in a patient who already received the FDA-approved singular-targeting CD19 CAR, Tisagenlecleucel, a decision was made with family and care team to pursue treatment on a phase I study of bispecific CD19/CD22 CAR T cells (NCT03241940). Eligibility criteria were met and the patient was enrolled on trial and proceeded with leukapheresis and CAR manufacturing.

Cell manufacturing was pursued using the closedsystem, semiautomated Miltenyi CliniMACs Prodigy

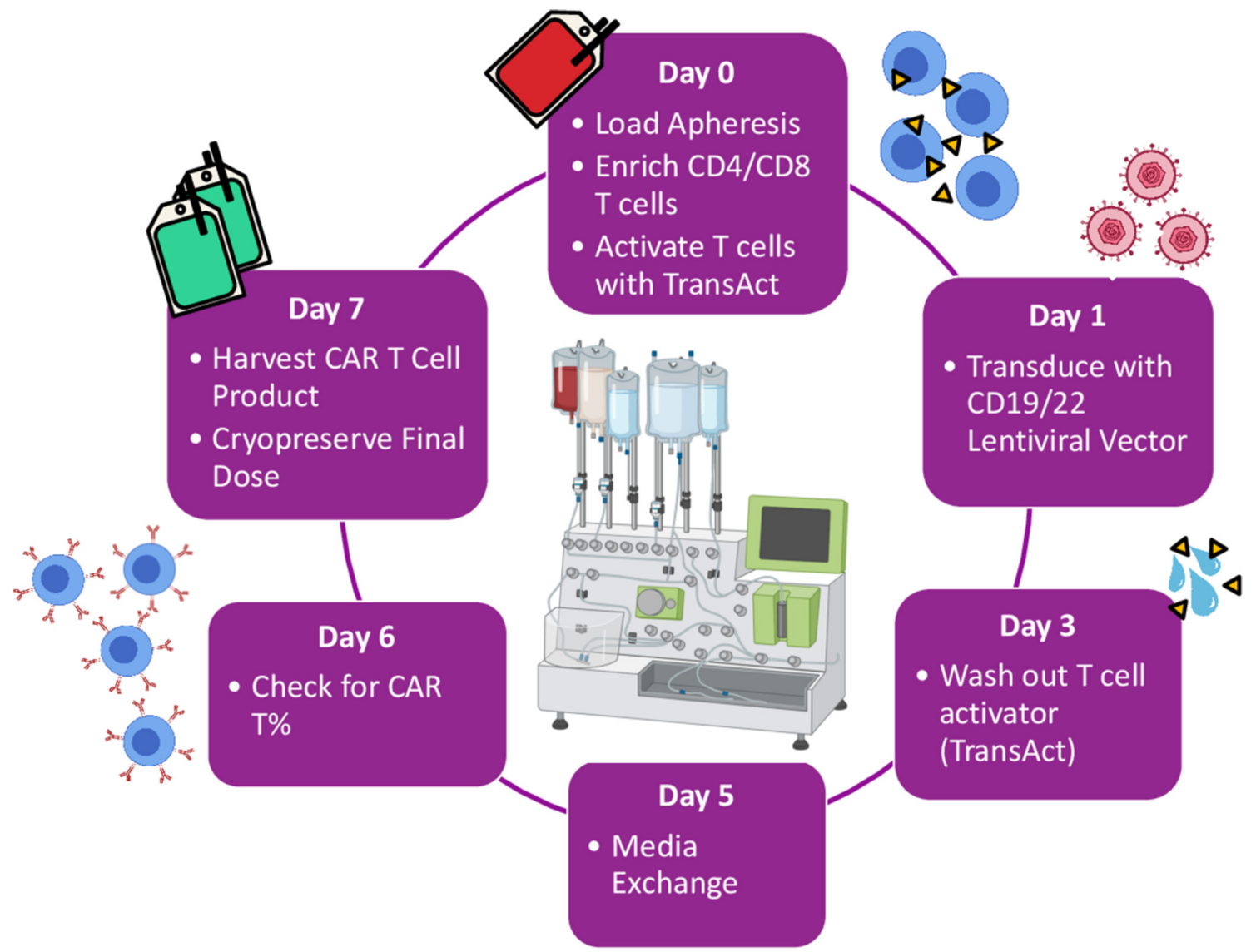

Figure 1 CD19/22 CAR T+ manufacturing schema. CD19/22 CAR T cells were manufactured using the Miltenyi CliniMACs Prodigy, a closed-system manufacturing platform. The manufacturing process details are specified in the methods section in Additional File 1. For this patient's CD19/22 CAR T+ cell product, the transduction efficiency was $48.12 \%$ and the vector copy number was 2, determined by qPCR-based detection on WHP post-transcriptional regulatory element. CAR, chimeric antigen receptor; PBMC, Peripheral blood mononuclear cells; WHP, Woodchuck. Hepatitis Virus 


\section{A Apheresis Sample}
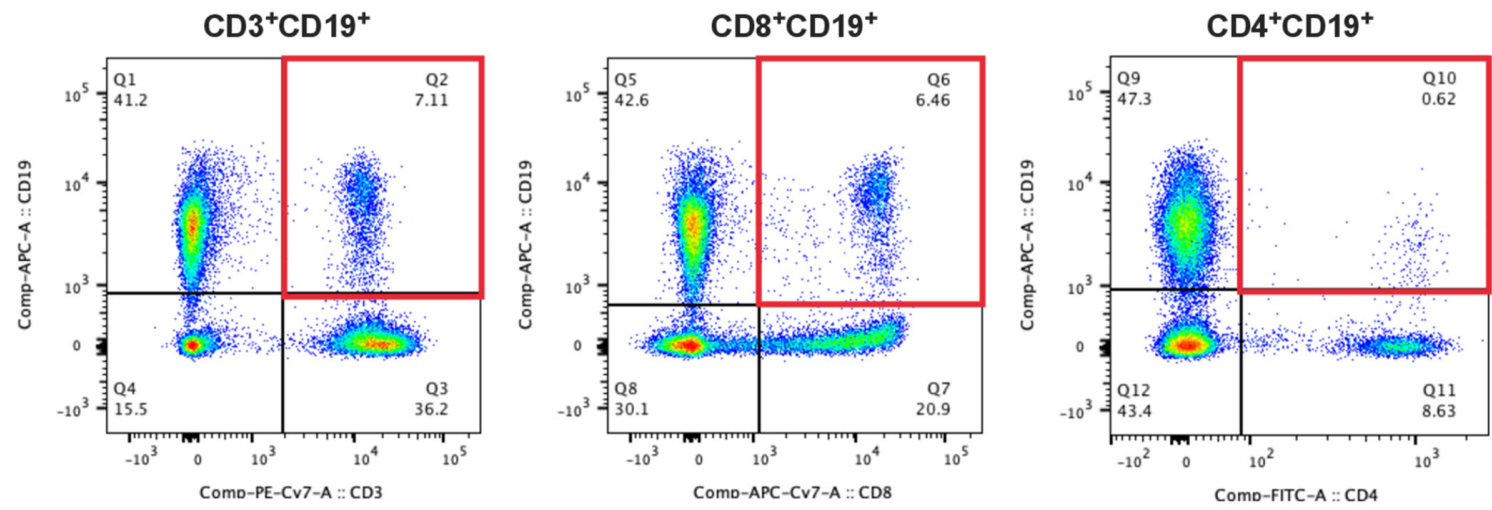

\section{B Enriched $\mathrm{CD}^{+} / \mathrm{CD}^{+}$Sample}
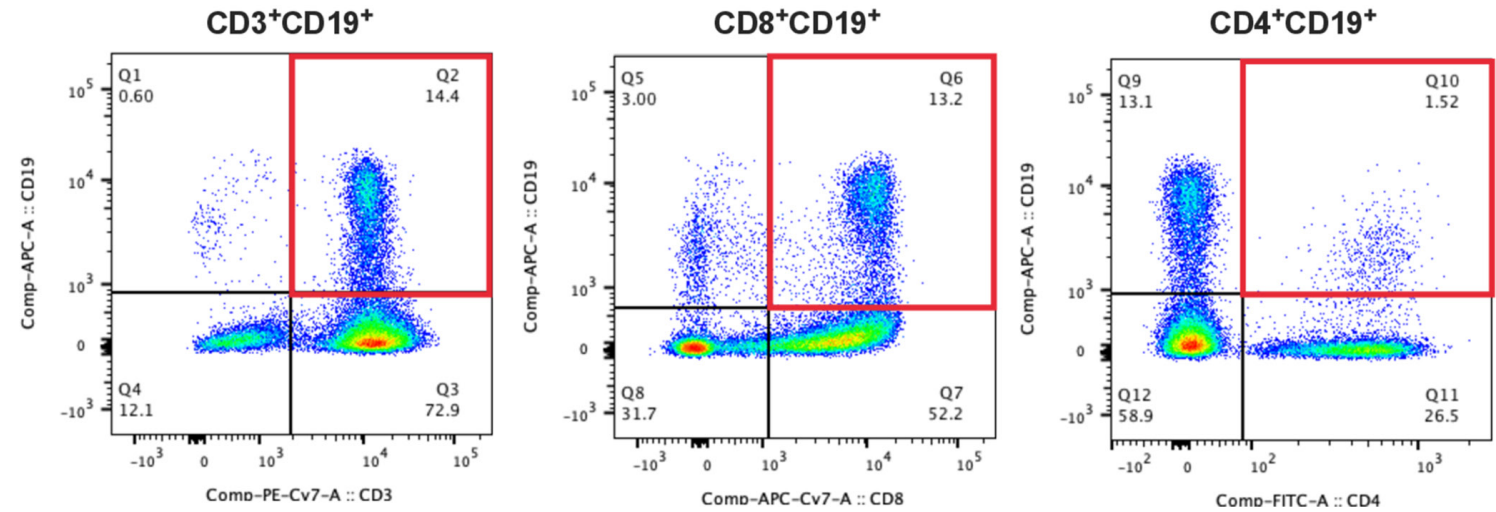

Figure 2 Phenotyping of apheresis and CD4+/CD8+ enriched samples. Patient apheresis and CD4+/CD8+ cells were analyzed by flow cytometry to phenotype cell populations pre-enrichment and postenrichment on the prodigy. The unusual CD3+CD19+ population $(7.1 \%)$ and specifically the CD8+CD19+ population (6.5\%) present in the apheresis $(A)$ was enriched during the CD4/CD8 positive selection (B), resulting in increases of both populations, to $14.4 \%$ and $13.2 \%$, respectively. The CD4+CD19+ population was minimal to begin with $(0.62 \%)$ and increased during enrichment $(1.52 \%)$. CAR, chimeric antigen receptor.

(see additional data for detailed methods). During production, as illustrated in the manufacturing schema (figure 1), T cells underwent a CD4 and CD8 positive bead selection to positively select $\mathrm{T}$ cells for transduction. Following CD4/CD8 T-cell selection, T-cell purity was assessed using flow cytometry and revealed an aberrant T-cell population expressing both CD3 and surface CD19 (figure 2). We hypothesized that these T cells likely represented the genetically engineered $\alpha \beta T$ cells (Rivocel) that were adoptively infused after HSCT and still circulating despite relapse. Flow cytometry confirmed the presence of this dual positive CD19+/CD3+ population in the leukapheresis product. Stimulation of the caspase pathway with titrated dosing of AP1903 (Rimiducid) effectively eliminated the target CD19+/CD3+ cell subsets in a dose-dependent manner, thus confirming that these cells are the iCas9-bearing $\alpha \beta$ T cells that were infused to the patient as part of his post-HSCT therapy (figure 3).

Given that CD19 is one of the target antigens of CAR, we additionally investigated the fate of the RivoCel as they underwent CD19-CAR transduction. The CD19+/ CD3+ population was not detected by flow cytometry (figure 4A) and iCas9 was not detected by PCR in the post-transduction product (figure 4B), which is consistent with elimination of CD19+ T cells by CD19-specific CAR+ $\mathrm{T}$ cells during expansion in culture. $\mathrm{T}$ cells dually positive for CD19 and for CD19-directed CARs were not detected, likely explained by in vitro fratricide. We excluded the possibility of CD19 masking by demonstrating absence of iCas9 by PCR in the post-transduction product. Presence of CD19+/CD3+ T cells in culture did not impact quality of manufacturing and the final CAR T-cell product met all manufacturing specifications with 100\% CD3 cells, 95\% viability, 48\% CAR transduction efficiency and successful expansion to meet the target dose of $3 \times 10^{6} \mathrm{CAR}$ T cells/ $\mathrm{kg}$. Although the product quality was not impacted, we did pause manufacturing and introduce production delay to further interrogate the nature and implications of this cell population.

\section{CONCLUSIONS}

With increasing sophistication in T-cell engineering techniques, clinical therapies for relapsed leukemia are broadening beyond traditional chemotherapy and bestmatched allogeneic stem cell transplantation. ${ }^{11}{ }^{12}$ CAR 
A

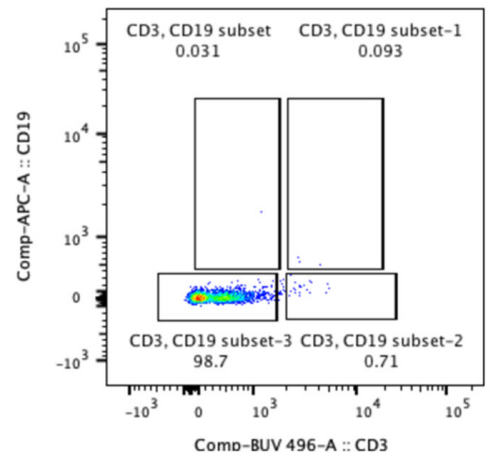

Placebo

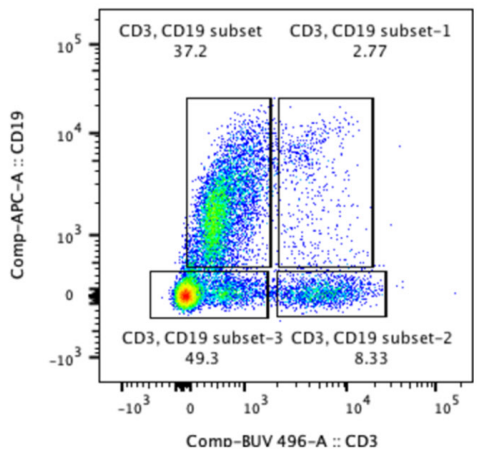

0.1 nM AP1903

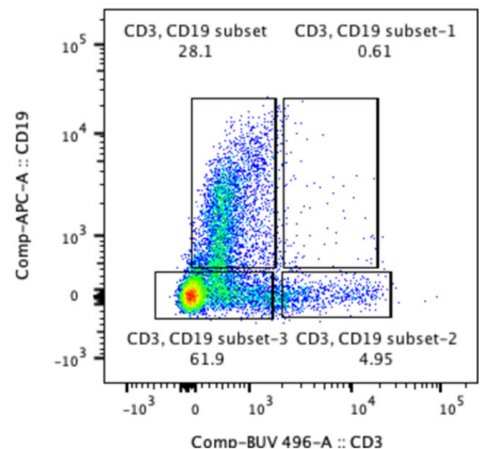

B

AP1903 Dose Titration

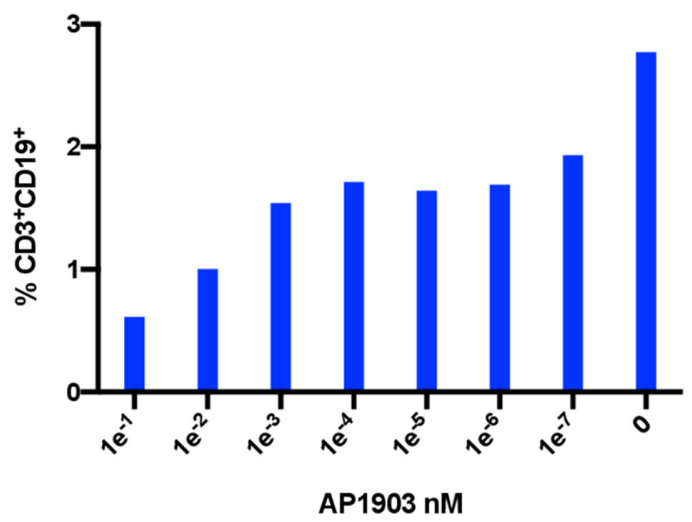

Figure 3 Rimiducid (AP1903) dose titration: 24-hour treatment of apheresis sample. Identity of the CD3+CD19+ population as the RivoCel $\alpha / \beta$ T cells containing the iCasp9 suicide gene was confirmed by rimiducid (AP1903) dose titration. PBMCs from the patient apheresis, following cryopreservation and thawing, were treated with placebo (excipient) versus AP1903 in a drug titration for 24 hours. Cryopreservation and thawing of this sample prior to analysis likely explains variability between the starting T-cell population in this figure as compared with figure 2, which was performed on fresh sample. A 78\% decrease in the CD3+ CD19+ T-cell subset is demonstrated following AP1903. Non-specific decrease in CD3+ CD19- and CD3-CD19+ cell subsets is additionally seen, however effect is preferential towards CD3+CD19+ T cells (A). AP1903 treatment resulted in a dose-dependent killing of the CD3+ CD19+ population (B). CAR, chimeric antigen receptor.

T-cell therapy has revolutionized our management of relapsed/refractory B-ALL. ${ }^{1-3} 1314$ With FDA-approval, CAR T cell use as a standard of care therapy has become increasingly accessible. Although CAR T cell therapy mediates durable remissions in some patients, with increasing clinical experience, we have seen that relapsed disease post-CAR challenges the curative potential of this strategy. ${ }^{215-17}$ Thus, CAR T cells can be used both as a bridging therapy prior to a consolidative stem cell transplant and as salvage therapy for post-transplant relapse. ${ }^{18}$

Recently, stem cell transplantation donor options have expanded to include haploidentical donors. ${ }^{19}$ Graft engineering strategies, including T-cell depletion, are employed to mitigate the risk of GvHD, in context of such immunogenetic disparity, and avoid the use of pharmacological immunosuppression. ${ }^{21}$ However, the elimination of $\mathrm{T}$ cells from the graft does come with an increased risk of infections and leukemia relapse. These hurdles can be overcome by combining a T cell-depleted transplant with an addback of $\alpha \beta$ T cells. ${ }^{22} \alpha \beta$ T cells have crucial properties in pathogen protection and graft-versus-leukemia
$(\mathrm{GvL})$ effect but they come with the additional risk of inducing severe GvHD. Therefore, strategies to promote GvL while sparing the risk of severe GvHD are needed. One such strategy is the use of $\mathrm{T}$ cells engineered to express caspase 9 (RivoCel), which can be activated via a dimerizing agent. In these engineered T cells, the caspase recruiting domain of the human caspase 9 was modified with a drug binding domain, allowing $\mathrm{T}$ cell elimination after administration of a chemical dimerization agent, AP1903. The administration of AP1903 dimerizes caspase 9 , causing activation which in turn further activates downstream caspases, leading to rapid apoptosis (minutes to hours).$^{893}$ In a recent multicenter USA (NCT03301168) and European Union (NCT02065869), prospective phase I-II clinical trial using $\alpha \beta$ haplo-HSCT followed by addback of RivoCel in patients with malignant or nonmalignant disorders RivoCel products were infused on day $14 \pm 4$ after the allograft. No post-transplant pharmacological GvHD prophylaxis was included in this study. Patients who developed GvHD resistant to conventional 
A

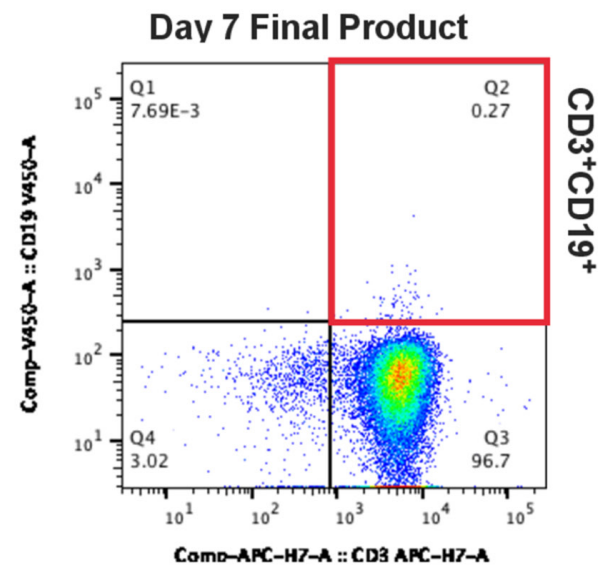

B

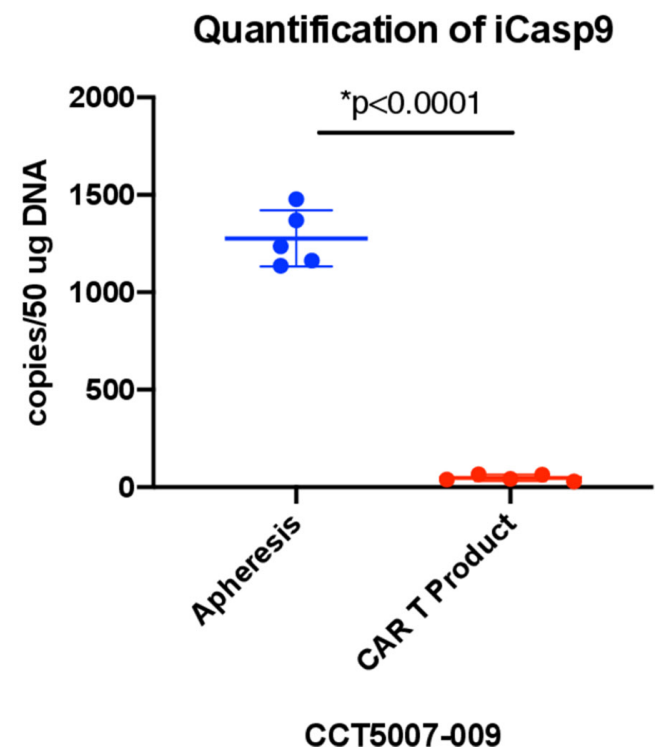

Figure 4 Absence of CD3+ CD19+ iCasp9+ Cells in CD19/22 CAR T+ product. Phenotyping of the final CD19/22 CAR T+ product by flow cytometry showed the absence of this CD3+CD19+ $\alpha / \beta$ T-cell population by day 7 (A). Reduction of the CD3+ CD19+ $\alpha / \beta$ T-cell population was confirmed by qPCR, detecting for the iCasp9 suicide gene (B). Copies of iCasp9 in the apheresis averaged 1276 copies/50 ng DNA, while the CAR product averaged 48 copies/50 ng DNA. Symbols indicate technical replicates. CAR, chimeric antigen receptor.

steroid therapy received up to three doses of AP1903 to activate iCas9. Preliminary results on a subset of patients with high-risk acute leukemias showed a 2-year LFS rate approaching $90 \%{ }^{24}$

As CAR T cell therapy and haploidentical stem cell transplantation are increasingly used in the clinical setting, we will likely see increasing frequency of patients receiving these manipulated cellular products in tandem. Here we describe the identification of a dual-expressing CD19+/CD3+ cell subset in an apheresis product undergoing CAR T-cell manufacturing derived from a patient previously treated with a haploidentical engineered stem cell product. Prior experience describes the dire outcome following inadvertent transduction of a CD19targeted CAR into a single leukemic CD19+ Bcell. The CAR masked CD19 expression on this CAR-expressing leukemic B cell, protecting it from CAR-mediated cytotoxicity and allowing it to develop into a fatal malignant clone. $^{25}$

Here, we explore the implications of having a CD19+ T-cell subset in a product undergoing CD19-specific CAR transduction and interrogated the origin of this population and its fate through CAR transduction. We utilized PCR to identify iCas9 gene within this population and used titration experiments to demonstrate elimination of this population following treatment with AP1903 (Rimiducid), thus confirming this population to be iCas9bearing engineered RivoCel. To investigate the fate of this population, we tracked this cell subset through CAR transduction using flow cytometry and gene sequencing. Using flow cytometry, we demonstrate elimination of this population following transduction with CD19-directed CARs, likely due to fratricide. To differentiate elimination of this population versus masking of CD19 by CD19directed CARs, we used PCR and demonstrated reduction of iCas9 sequence, corresponding to frank elimination of this subset following CAR transduction.

Although the CD19+ RivoCel population was eliminated from the T-cell product during CAR manufacturing, patient samples demonstrated persistence of circulating RivoCel at the time of relapse posthaploidentical HSCT, at a level of $10 \%$ of his CD3+ T cells (see Additional File 2; online supplemental figure 1). The clinical impact of circulating CD19+ T cells in a patient receiving CD19-specific CAR $\mathrm{T}$ cells remains unclear. The resultant increase in CD19 antigen load raises the possibility of increased CAR T-cell activation and concern for increased toxicity. Increased antigen load may additionally have beneficial effect in promoting CAR T-cell expansion and persistence with the sum effect likely individualized, dependent on patient factors including disease burden, baseline antigen load and toxicity risk. There were additional clinical implications as identification of this population introduced manufacturing delays while we investigated the nature of this cell population. This patient ultimately developed progressive leukemia with disseminated fungal infection and renal toxicity, precluding treatment on trial, but we found the identification of this CD19+/CD3+ cell population in the apheresis product and the fate of this population spanning CAR manufacturing notable, as this has yet to be described in the literature.

In summary, we report the identification of CD19+/ CD3+ $\mathrm{T}$ cells in an apheresis product undergoing CAR transduction derived from a patient previously treated with a haploidentical transplant and an engineered $\alpha \beta$ 
addback product modified to express truncated CD19 as a marker of this population (RivoCel). Although Rivocel $\mathrm{T}$ cells were eliminated during CD19-CAR manufacturing, this synthetic CD19-expressing T-cell population persisted in the patient at the time of relapse and CAR work-up. With CD19-directed therapies taking on a pivotal role in managing patients who relapse poststem cell transplantation, we suggest consideration in using alternative markers to CD19 as a synthetic identifiers for post-transplant addback products, as CD19-expression on effector $\mathrm{T}$ cells may impact subsequent treatment using CD19-directed therapy.

Contributors LS was responsible for experimental design, patient clinical care and was primary author of the manuscript. SP performed the bulk of experimentation, designed the figures and legends and was involved in writing the manuscript. $A B$ was involved in performing experiments and writing the manuscript. $S R, K D$ and RGM were involved in patient clinical care and experimental design. $C B$ and CE were involved in patient clinical care. NB and J0 were involved in conducting experiments and figure generation. CM and SF supervised the project. All authors read and approved the final manuscript.

Funding The authors have not declared a specific grant for this research from any funding agency in the public, commercial or not-for-profit sectors.

Competing interests RGM is a consultant for Lyell Immunopharma, Xyphos and GammaDelta Therapeutics. Other authors declare that they have no competing interests.

\section{Patient consent for publication Not required.}

Ethics approval Subject was enrolled and consented on a phase I study of safety and feasibility of CD19/CD22 Bispecific CAR T cells (NCT03241940). Protocol was reviewed and approved by Stanford IRB Administrative Panels in Human Subjects in Medical Research ('Stanford IRB'). FWA00000935, IRB00000348.

Provenance and peer review Not commissioned; externally peer reviewed.

Open access This is an open access article distributed in accordance with the Creative Commons Attribution Non Commercial (CC BY-NC 4.0) license, which permits others to distribute, remix, adapt, build upon this work non-commercially, and license their derivative works on different terms, provided the original work is properly cited, appropriate credit is given, any changes made indicated, and the use is non-commercial. See http://creativecommons.org/licenses/by-nc/4.0/.

\section{ORCID iD}

Liora Schultz http://orcid.org/0000-0001-9512-7597

\section{REFERENCES}

1 Lee DW, Kochenderfer JN, Stetler-Stevenson M, et al. T cells expressing CD19 chimeric antigen receptors for acute lymphoblastic leukaemia in children and young adults: a phase 1 dose-escalation trial. Lancet 2015;385:517-28.

2 Maude SL, Laetsch TW, Buechner J, et al. Tisagenlecleucel in children and young adults with B-cell lymphoblastic leukemia. $N$ Engl $J$ Med 2018;378:439-48.

3 Park JH, Rivière I, Gonen M, et al. Long-Term follow-up of CD19 CAR therapy in acute lymphoblastic leukemia. N Engl J Med 2018;378:449-59.

4 Gardner RA, Finney O, Annesley C, et al. Intent-To-Treat leukemia remission by CD19 CAR T cells of defined formulation and dose in children and young adults. Blood 2017;129:3322-31.
5 Maude SL, Frey N, Shaw PA, et al. Chimeric antigen receptor T cells for sustained remissions in leukemia. N Engl J Med 2014;371:1507-17.

6 Klebanoff CA, Gattinoni L, Restifo NP. Sorting through subsets: which T-cell populations mediate highly effective adoptive immunotherapy? J Immunother 2012;35:651-60.

7 Hinrichs CS, Borman ZA, Cassard L, et al. Adoptively transferred effector cells derived from naive rather than central memory CD8+ T cells mediate superior antitumor immunity. Proc Natl Acad Sci U S A 2009;106:17469-74.

8 Zhou X, Dotti G, Krance RA, et al. Inducible caspase-9 suicide gene controls adverse effects from alloreplete $T$ cells after haploidentical stem cell transplantation. Blood 2015;125:4103-13.

9 Di Stasi A, Tey S-K, Dotti G, et al. Inducible apoptosis as a safety switch for adoptive cell therapy. N Engl J Med 2011;365:1673-83.

10 Tey S-K, Dotti G, Rooney CM, et al. Inducible caspase 9 suicide gene to improve the safety of allodepleted $\mathrm{T}$ cells after haploidentical stem cell transplantation. Biol Blood Marrow Transplant 2007;13:913-24.

11 Bertaina A, Roncarolo MG, Engineering G. Graft engineering and adoptive immunotherapy: new approaches to promote immune tolerance after hematopoietic stem cell transplantation. Front Immunol 2019;10:1342.

12 Sadelain M, Brentjens R, Rivière I. The basic principles of chimeric antigen receptor design. Cancer Discov 2013;3:388-98.

13 Kalos M, Levine BL, Porter DL, et al. T cells with chimeric antigen receptors have potent antitumor effects and can establish memory in patients with advanced leukemia. Sci Trans/ Med 2011;3:95ra73.

14 Brentjens RJ, Davila ML, Riviere I, et al. CD19-targeted T cells rapidly induce molecular remissions in adults with chemotherapyrefractory acute lymphoblastic leukemia. Sci Trans/ Med 2013;5:177ra38

15 Majzner RG, Mackall CL. Tumor antigen escape from CAR T-cell therapy. Cancer Discov 2018;8:1219-26.

16 Sotillo E, Barrett DM, Black KL, et al. Convergence of acquired mutations and alternative splicing of CD19 enables resistance to CART-19 immunotherapy. Cancer Discov 2015;5:1282-95.

17 Schultz L, Gardner R. Mechanisms of and approaches to overcoming resistance to immunotherapy. Hematology Am Soc Hematol Educ Program 2019;2019:226-32.

18 Jacoby $\mathrm{E}$. The role of allogeneic HSCT after CAR T cells for acute lymphoblastic leukemia. Bone Marrow Transplant 2019;54:810-4.

19 Handgretinger R, Klingebiel T, Lang P, et al. Megadose transplantation of purified peripheral blood CD34(+) progenitor cells from HLA-mismatched parental donors in children. Bone Marrow Transplant 2001;27:777-83

20 Kanakry CG, Fuchs EJ, Luznik L. Modern approaches to HLAhaploidentical blood or marrow transplantation. Nat Rev Clin Oncol 2016;13:132.

21 Pierini A, Ruggeri L, Mancusi A, et al. T cell depletion and NO post transplant immune suppression allow separation of graft versus leukemia from graft versus host disease. Bone Marrow Transplant 2019:54:775-9.

22 Ruggeri A, Merli P, Algeri M, et al. Comparative Analysis of AlphaBeta T-Cell and B-Cell Depleted (abTCD) HLA-Haploidentical Hematopoietic Stem Cell Transplantation (haplo-HSCT) Versus Abtcd Haplo-HSCT with T-Cell Add-Back of Rivogenlecleucel Cell [Donor T Cells Transduced with the Inducible Caspase 9 (iC9) Gene Safety Switch] in Children with High-Risk Acute Leukemia (AL) in Remission. Blood 2019;134:145.

23 Zhou X, Naik S, Dakhova O, et al. Serial activation of the inducible caspase 9 safety switch after human stem cell transplantation. Mol Ther 2016;24:823-31.

24 Locatelli F, Ruggeri A, Merli P, et al. Administration of BPX-501 cells following A $\beta$ T and B-Cell-Depleted HLA haploidentical HSCT (haploHSCT) in children with acute leukemias. Blood 2018;132:166.

25 Ruella M, Xu J, Barrett DM, et al. Induction of resistance to chimeric antigen receptor $\mathrm{T}$ cell therapy by transduction of a single leukemic $B$ cell. Nat Med 2018;24:1499-503. 